\title{
Research Article \\ Investigation of Baryons in the Hypercentral Quark Model
}

\author{
N. Tazimi $\mathbb{D}$ and P. Sadeghi Alavijeh \\ Department of Physics, University of Kashan, Kashan, Iran \\ Correspondence should be addressed to N. Tazimi; nt_physics@yahoo.com
}

Received 19 June 2021; Revised 4 September 2021; Accepted 15 September 2021; Published 21 October 2021

Academic Editor: Enrico Lunghi

Copyright (c) 2021 N. Tazimi and P. Sadeghi Alavijeh. This is an open access article distributed under the Creative Commons Attribution License, which permits unrestricted use, distribution, and reproduction in any medium, provided the original work is properly cited. The publication of this article was funded by $\mathrm{SCOAP}^{3}$.

In the present study, we consider baryons as three-body bound systems according to the hypercentral constituent quark model in configuration space and solve the three-body Klein-Gordon equation. Then, we analyze perturbative spin-dependent and isospindependent interaction effects. To find the analytical solution, we use screened potential and calculate the eigenfunctions and eigenvalues of some baryons. We consider exclusive semileptonic decays of bottom and charm baryons and apply the differential decay width with the Isgur-Wise function and arrive at the rates for some semileptonic baryon decays. The results prove more enhanced compared to recent works and comply well with the experimental data.

\section{Introduction}

Exact solutions have been of paramount importance in the study of relativistic and nonrelativistic quantum systems as they can yield useful information on these systems. Nevertheless, there are so few exact solutions that approximate methods are often used to investigate quantum systems. For instance, Schrödinger, Klein-Gordon, and BetheSalpeter equations have been solved through proper approximation [1-3] to the centrifugal term for a number of potentials like Eckart [4], Manning-Rosen [5], Pusch-Terrel [6], Hulthen [7], and Wei et al. [8] employed an approximation to arrive at the approximative analytical l-wave scattering solutions of the Schrödinger equation. Their findings closely comply with other studies as for short-range potentials but differ as for long-range ones. They also solved the KleinGordon equation by the unequal scalar and vector Hulthen potential via a creative approximation to the centrifugal term [9].

In this study, the baryons are treated as spinindependent three-quark systems and based on hyperspherical approach; a nonrelativistic quark model is presented for them. Considering the significance of the analytical solution for the qualitative analysis of the spectrum, we present analytical formalisms for wave functions and energy levels by using the screened potential. In the 1980s, a screening mul- tiplicative factor in the potential was presented $[10,11]$. Accordingly for larger distances, the screened linear term in the potential acts as the dominant term, so the most excited states are supposed as confinement states, which are determined by considering merely the screened linear interaction. Also for short distances, due to the small linear term, the Coulomb term is dominant. Therefore, screened potential accounts for the behavior of quarks according to the QCD rules [12]. Finally, we make use of spin-isospin and flavor-dependent interactions to identify the baryon energy spectrum more accurately.

In the next section of this paper, we examine the heavyto-light semileptonic decays of heavy baryons. These decays are utilized to determine the Cabibbo-Kobayashi-Maskawa (CKM) matrix elements. For this purpose, we use the Isgur-Wise function (IWF). The IWF has provided physicists with remarkable results for transitions of bottom baryons into charm baryons [13]. The calculation of the IWF yields useful information on the decay width, branching ratio, and CKM matrix $[10,14,15]$. Ivanov et al. studied the semileptonic decays of bottom and charm baryons within the relativistic quark model [16]. Recent measurements and the pertinent theoretical approaches contribute greatly to understanding the decays of bottom baryons. A number of scholars have made attempts to study the structure of heavy-baryon transitions via IWF formalism. Ke 
et al. [17] investigated baryon weak decays in the light-front quark model. Using the heavy diquark model for baryons, Viet described the matrix elements of weak currents of baryons through universal IWF [18]. Ebert et al. [19] used the relativistic quark model to calculate the semileptonic decay rates of heavy baryons. Cardarelli and Simula explored the corresponding form factors of heavy baryons in a lightfront constituent quark model [20]. Körner et al. also studied the heavy baryon transitions and analyzed the decay rates, polarization effects, lifetimes and angular decay distributions [21].

In our previous works, we solved the Schrödinger equation for different potentials for few-quark systems [22-25]. In a more recent work, we investigated the relativistic Klein-Gordon equation analytically for the Deng-Fan potential and Hulthen plus Eckart potential under the equal vector and scalar potential conditions [26]. In this paper, we study our three-body system and make use of the hyperspherical coordinates and calculate the parameters of the IWF for some baryons. The purpose of this study is to investigate the heavy-baryon decays in hyperspherical coordinates. To this end, we use the hyperradial Klein-Gordon equation in configuration space in Section 2. Afterwards, in Section 3, we solve the Klein-Gordon equation via screened potential. Then, by analyzing the IWF corresponding to hyperspherical coordinates, we study the heavy-baryon decays and calculate the decay width of these particles in Section 4. And finally, the relevant conclusions are presented in Section 5.

\section{The Three-Body Problem}

Calculations of mass and energy of some heavy baryons have been carried out through a simple semirelativistic quark model. Assuming a dominant SU(6)-invariant spectrum in the nonrelativistic hypercentral constituent quark model which led to the apparent success in describing the mass spectra and other observables motivates us to introduce a spin-independent relativistic description to approximate the problem and then deal the SU(6)-breaking interactions perturbatively.

In this study, we investigate baryons using a semirelativistic model based on the hypercentral quark constituent model. Due to the significance of the relativistic analysis of particles behavior within bound systems, we study baryons as three-quark systems while taking relativistic effects into account. Totally relativistic study of baryons demands solving the Dirac equation for each quark (while considering interquark interactions). Totally relativistic solution to a three-body problem is difficult. Thus, we have to apply a few simplifications to solve this problem. As the first step to simplify the particles' spin and isospin, we introduce spin and isospin effects perturbatively. The reason for the assumption is the experimental baryon spectra has a SU(6) symmetry for baryon resonances. Therefore, for the SU(6)invariance part, we can considerations as a three-quark system of zero-spin and use a separate relativistic description of spin for the baryon spectrum. The exact analytical formulas for energy levels and wave functions have been obtained, which can be used for a parametric analysis of the problem.
The three-body Klein-Gordon equation with equal scalar and vector potentials in the natural unit $h=c=1$ is of this form [9]:

$$
\left(\sum_{i=1}^{3} p_{i}^{2}+(m+\varepsilon) \sum_{j \neq i}^{3} V_{i j}+3\left(m^{2}-\varepsilon^{2}\right)\right)|\psi\rangle=0
$$

where $|\psi\rangle$ denotes the total wave function of the three-body system and $3 \varepsilon$ is its total energy. $V_{i j}$ stands for the potential of interaction between the $i$-th and $j$-th particles. We solve the three-body spherical coordinates system and express the spatial degrees of freedom in terms of Jacobian momentums. Jacobi coordinates to define three-body basis states are given by $[27,28]$

$$
\begin{gathered}
\rho=\frac{r_{1}-r_{2}}{\sqrt{2}}, \\
\lambda=\frac{1}{\sqrt{6}}\left(r_{1}+r_{2}-2 r_{3}\right) .
\end{gathered}
$$

Here $r_{i}(i=1,2,3)$ denote coordinate of the $i$-th constituent quark and $m$ is the constituent quark mass. In the numerical calculations we take the values of the quark masses as $m_{s}=0.483 \mathrm{GeV}, \quad m_{u}=m_{d}=0.336 \mathrm{GeV}, \quad m_{b}=$ $4.950 \mathrm{GeV}$, and $m_{c}=1.550 \mathrm{GeV}$. In hyperspherical coordinates, the hyperradius and hyperangle are defined as in Figure 1.

$$
x=\sqrt{\rho_{i}^{2}+\lambda_{i}^{2}}=\left[\frac{r_{12}^{2}+r_{23}^{2}+r_{13}^{2}}{3}\right]^{1 / 2}, \varphi=\arctan \left(\frac{\rho_{i}}{\lambda_{i}}\right) .
$$

In addition to $x$ and $\varphi$, four polar angles $\Omega_{\rho l}$ and $\Omega_{\lambda l}$ show the orientation of Jacobian vectors according to reference experimental frameworks. For Jacobian momentums, $P=\left(p_{1}+p_{2}+p_{3}\right) / \sqrt{3}$. In mass center framework, $p=0$. Accordingly, $\sum_{i=1}^{3} p_{i}^{2}$ for three particles in six dimensions is

$$
\sum_{i=1}^{3} p_{i}^{2}=p_{\rho l}^{2}+p_{\lambda l}^{2}=-\left(\nabla_{\rho l}^{2}+\nabla_{\lambda l}^{2}\right)=\nabla_{\xi_{i}}^{2}
$$

In hyperspherical coordinates in the $D$-dimensional space, the Laplace operator for $N$ particles generally becomes

$$
\begin{aligned}
\sum_{i=1}^{N-1} \nabla_{\xi_{i}}^{2} & =-\left(\frac{d^{2}}{d x^{2}}+\frac{D-1}{x} \frac{d}{d x}+\frac{L^{2}(\Omega)}{x^{2}}\right) \\
& =-\left(\frac{1}{x^{D-1}}\left[\frac{d}{d x} x^{D-1} \frac{d}{d x}\right]+\frac{L^{2}(\Omega)}{x^{2}}\right) .
\end{aligned}
$$

The term $L^{2}(\Omega) / x^{2}$, as a generalization of the centrifugal barrier for the six-dimensional space, involves the angular coordinates $\Omega_{\rho}$ and $\Omega_{\lambda}$ and the hyperangle $\xi$. In a three- 


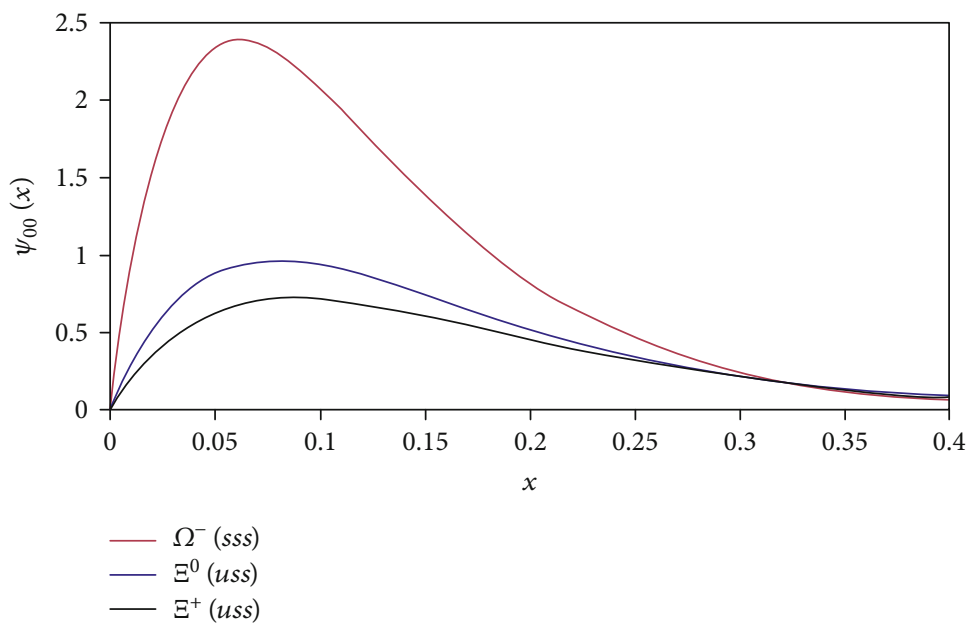

FIgURE 1: The hyperradius and hyperangle.

body system,

$$
L^{2}\left(\Omega_{5}\right) Y_{[\gamma]}\left(\Omega_{5}\right)=\gamma(\gamma+4) Y_{[\gamma]}\left(\Omega_{5}\right)
$$

where $\lambda$ is the polynomial order and $\lambda=2 n+l_{\rho}+l_{\lambda} \cdot l_{\rho}\left(l_{\lambda}\right)$ where $n$ is a positive integer and $l_{\rho}$ and $l_{\lambda}$ denote the quantum numbers of angular momentums related to $\lambda$ and $\rho$ coordinates. According to the hypercentral hypothesis, the interacting potential in equation (1) can be expressed as a function of hyperradius $x$ only:

$$
\begin{aligned}
V\left(\left|r_{1}-r_{2}\right|\right)+ & V\left(\left|r_{2}-r_{3}\right|\right)+V\left(\left|r_{1}-r_{3}\right|\right) \\
& \approx V\left(\left|r_{1}-r_{2}\right|,\left|r_{2}-r_{3}\right|,\left|r_{1}-r_{3}\right|\right) \approx V(x) .
\end{aligned}
$$

The potential $V(x)$ is known as hypercentral, which is invariant for any rotation in $D$-dimensional space spanned by the coordinates and $O(D)$ symmetry. Depending on the hyperradius $x$ implies that the potential has a three-body character as the dependence on the single-pair coordinates cannot be disentangled from the other particles. Accordingly, the following three-body Klein-Gordon equation,

$$
\left[\frac{\partial^{2}}{\partial x^{2}}+\frac{5}{x} \frac{\partial}{\partial x}-\frac{\gamma(\gamma+4)}{x^{2}}+3\left(\varepsilon^{2}-m^{2}\right)-(\varepsilon+m) V(x)\right] \psi_{\nu, \gamma}(x)=0
$$

can be reduced to a six-dimensional time-dependent Schrodinger-like equation:

$$
\left(H_{0}+V\right) \psi=E \psi
$$

where

$H_{0}=\frac{\partial^{2}}{\partial x^{2}}+\frac{5}{x} \frac{\partial}{\partial x}-\frac{\gamma(\gamma+4)}{x^{2}}, V=-(m+\varepsilon) V(x), E=-3\left(\varepsilon^{2}-m^{2}\right)$.

\section{Screened Potential}

Screened potential is of the following form [12]:

$$
V(x)=\bar{\sigma} x\left(\frac{1-e^{-\mu x}}{\mu x}\right)-\frac{2}{3} \bar{\alpha}_{s} \frac{e^{-\mu x}}{x} .
$$

The hat over the parameters in the potential distinguishes them from the nonscreened case; $\bar{\sigma}$ corresponds to the string tension of the confinement in screened case. $\bar{\alpha}_{s}$ is a parameter representing the Coulomb strength, and $\mu$ stands for a screening parameter. By using the following approximation to the first order,

$$
e^{-\mu x} \simeq 1-\mu x+\frac{(\mu x)^{2}}{2 !}-\frac{(\mu x)^{3}}{3 !}+\cdots
$$

This potential turns out as

$$
V(x)=\bar{\sigma} x-\frac{2}{3} \frac{\bar{\alpha}_{s}}{x}+\frac{2}{3} \bar{\alpha}_{s} \mu .
$$

By using variable changes $s=(1 / x), \beta=1 / x_{0}$, and $z=s$ $-\beta$,

$$
\begin{aligned}
x & =\frac{1}{s}=\frac{1}{z+\beta}=\frac{1}{\beta}\left(1-\frac{z}{\beta}+\left(\frac{z}{\beta}\right)^{2}+\cdots\right) \\
& =\frac{1}{\beta}\left(1-\frac{s-\beta}{\beta}+\left(\frac{s-\beta}{\beta}\right)^{2}+\cdots\right) .
\end{aligned}
$$


By simplifying the above equation, we arrive at

$$
x=\frac{1}{\beta}\left(3-\frac{3 s}{\beta}+\frac{s^{2}}{\beta^{2}}\right)=3 x_{0}-\frac{3 x_{0}^{2}}{x}+\frac{x_{0}^{3}}{x^{2}} .
$$

$x_{0}$ is supposed as a characteristic radius of the baryon. The screened potential appears as

$$
\begin{gathered}
V(x)=3 \bar{\sigma} x_{0}-\frac{3 \bar{\sigma} x_{0}^{2}+2 / 3 \bar{\alpha}_{s}}{x}+\frac{\bar{\sigma} x_{0}^{3}}{x^{2}}+\frac{2}{3} \bar{\alpha}_{s} \mu \\
{\left[\frac{\partial^{2}}{\partial x^{2}}+\frac{5}{x} \frac{\partial}{\partial x}-\frac{\gamma(\gamma+4)}{x^{2}}+3\left(\varepsilon^{2}-m^{2}\right)-(\varepsilon+m)\right.} \\
\left.\cdot\left(3 \bar{\sigma} x_{0}-\frac{3 \bar{\sigma} x_{0}^{2}+(2 / 3) \bar{\alpha}_{s}}{x}+\frac{\bar{\sigma} x_{0}^{3}}{x^{2}}+\frac{2}{3} \bar{\alpha}_{s} \mu\right)\right] \psi_{v, \gamma}(x)=0
\end{gathered}
$$

which can be reformulated as

$$
\left[\frac{\partial^{2}}{\partial x^{2}}+\frac{5}{x} \frac{\partial}{\partial x}+\left(-A+\frac{B}{x}-\frac{C}{x^{2}}\right)\right] \psi_{\nu, \gamma}(x)=0,
$$

where $A, B$, and $C$ appear as the following.

$$
\begin{gathered}
A=(\varepsilon+m)\left[-3(\varepsilon-m)+3 \bar{\sigma} x_{0}+\frac{2}{3} \bar{\alpha}_{s} \mu\right], \\
B=(\varepsilon+m)\left(3 \bar{\sigma} x_{0}^{2}+\frac{2}{3} \bar{\alpha}_{s}\right), \\
C=\gamma(\gamma+4)+(\varepsilon+m) \bar{\sigma} x_{0}^{3} .
\end{gathered}
$$

By using Nikiforov-Uvarov (NU) method (see appendix), we calculate the values for constant parameters $\alpha_{i} s$, as we calculated these parameters in [26]. They are shown in Table 1. By introducing these values into equation (18),

$$
\begin{gathered}
(2 v+1) \sqrt{A}-B+2 \sqrt{A} \times \sqrt{4+C}=0 \Rightarrow A \\
=\left(\frac{B}{2 v+1+2 \sqrt{4+C}}\right)^{2} .
\end{gathered}
$$
$(17)$

By introducing the values of $A, B$, and $C$ into equation

$$
-3(\varepsilon-m)+3 \bar{\sigma} x_{0}+\frac{2}{3} \bar{\alpha}_{s} \mu=\frac{(\varepsilon+m)\left(3 \bar{\sigma} x_{0}^{2}+(2 / 3) \bar{\alpha}_{s}\right)^{2}}{\left(2 v+1+2 \sqrt{(\gamma+2)^{2}+(\varepsilon+m) \tilde{\sigma} x_{0}^{3}}\right)^{2}} .
$$

And new variables are defined as

$$
\begin{gathered}
\tilde{\sigma}=3 \bar{\sigma} x_{0}^{2}+\frac{2}{3} \bar{\alpha}_{s}, \\
\tilde{\gamma}+2=\left[(\gamma+2)^{2}+(\varepsilon+m) \bar{\sigma} x_{0}^{3}\right]^{1 / 2} .
\end{gathered}
$$

TABLE 1: Constant parameters.

\begin{tabular}{lc}
\hline Analytical value & Constant \\
\hline 5 & $\alpha_{1}$ \\
0 & $\alpha_{2}$ \\
0 & $\alpha_{3}$ \\
-2 & $\alpha_{4}$ \\
0 & $\alpha_{5}$ \\
$A$ & $\alpha_{6}$ \\
$-B$ & $\alpha_{7}$ \\
$4+C$ & $\alpha_{8}$ \\
$A$ & $\alpha_{9}$ \\
$1+2 \sqrt{4+C}$ & $\alpha_{10}$ \\
$2 \sqrt{A}$ & $\alpha_{11}$ \\
$-2+\sqrt{4+C}$ & $\alpha_{12}$ \\
$-\sqrt{A}$ & $\alpha_{13}$ \\
\hline
\end{tabular}

With the above variable changes and simple calculations employed, the energy eigenvalues will be

$$
E_{v, \gamma}=\frac{2}{3} \bar{\alpha}_{s} \mu+3 \bar{\sigma} x_{0}-\frac{m \tilde{\sigma}^{2}}{2(\nu+\tilde{\gamma}+5 / 2)^{2}}
$$

As $\alpha_{3}=0$, then to calculate the wave function, the Logger polynomial is used. Therefore, the wave function will be

$$
\psi_{v, \gamma}(x)=N_{v, \gamma} x^{\tilde{\gamma}} e^{-g x} L_{v}^{2 \tilde{\gamma}+4}(2 g x) .
$$

Here, $\gamma$, the degree of the polynomial, is called the grand orbital quantum number and is equal to $2 n+l_{\rho}+l_{\gamma}$ and $v$ $=0,1,2, \cdots$ according to the hypercentral hypothesis. Also, $g=(2 / 3) \alpha_{s}(m+\varepsilon) /(\nu+\tilde{\gamma}+5 / 2)$ and $N_{\nu, \gamma}$ is a normalization coefficient. The baryon masses are obtained via

$$
N_{\nu, \gamma}=\left[\frac{(\nu !)(2 g)^{(2 \tilde{\gamma}+6)}}{2(\nu+\tilde{\gamma}+5 / 2)(\nu+2 \tilde{\gamma}+4) !}\right]^{1 / 2} .
$$

Similarly, we can repeat this iteration for other modes; we obtain the energy and the corresponding wave function for any arbitrary $v$. We have plotted the distribution of hyperradial wave function density for $\Omega^{-}, \Xi^{0}, \Xi^{+}$in Figure 2. As expected, the wave function peak height grows larger as the baryon mass increases. By obtaining energy, we calculate the mass of baryon according to the following equation:

$$
M_{v, \gamma}=E_{0}+E_{v, \gamma}+\left\langle H_{\mathrm{int}}\right\rangle
$$

where $E_{0}$ is calculated for the ground state. $H_{\text {int }}$ is the perturbation Hamiltonian and depends on the spin, isospin, and flavor degrees of freedom. Table 2 displays the fitted values for calculating the energy eigenvalue. 


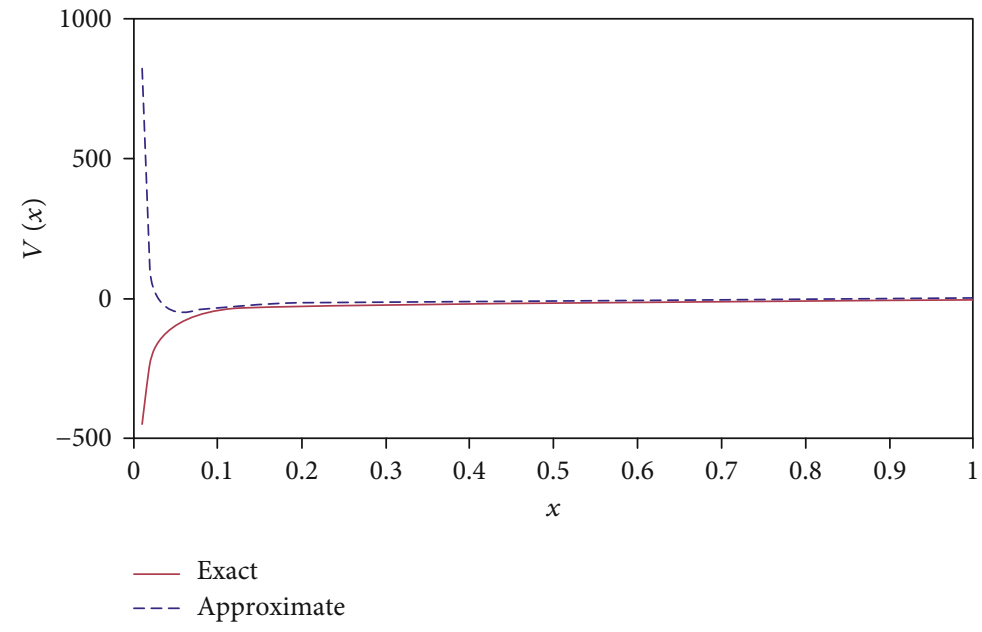

Figure 2: Wave function of baryons.

TABLE 2: Fitted values of the potential.

\begin{tabular}{lc}
\hline Parameter & Value \\
\hline $\bar{\alpha}_{s}$ & 6.78 \\
$\mu\left(\mathrm{fm}^{-1}\right)$ & 0.15 \\
$\bar{\sigma}\left(\mathrm{fm}^{-2}\right)$ & 1.08 \\
\hline
\end{tabular}

Table 3 presents the results obtained for spinless ground state of some baryons. These results are consistent with particle data group [29]. Even though the terms of tensor and spin-orbit are small, to achieve higher accuracy, these terms are considered as perturbation potentials and the perturbation energy levels are calculated. The perturbation method is helpful when the eigenstates cannot be obtained easily. To obtain the first-order correction of the energy eigenstates, we calculate the expected value of the perturbed potential while the system is in the unperturbed state.

$$
\left\langle H_{\mathrm{int}}(r)\right\rangle=\int \psi_{v, \gamma}^{*}(r) H_{\mathrm{int}}(r) \psi_{v, \gamma}(r) r^{2} d r
$$

where $H_{\text {int }}(r)=H_{S_{1}, S_{2}}(r)+H_{I_{1}, I_{2}}(r)+H_{S, I}(r)$. This interaction for a particle by total spin $S$ and isospin $T$ is of the following form:

$H_{\text {int }}(r)=\left[A_{S}\left(S^{2}-\frac{9}{4}\right)+A_{I}\left(T^{2}-\frac{9}{4}\right)+A_{S I}\left(S^{2}-\frac{9}{4}\right)\left(T^{2}-\frac{9}{4}\right)\right] \frac{e^{-\mu r}}{r}$,

where $\quad A_{S}=35.46, A_{I}=33.49, A_{S I}=-3.94 \quad$ (all in $\mathrm{MeV} \cdot \mathrm{fm}$ ) and $S$ is spin and $T$ is isospin of the baryon. From equation (28), it can be deduced that $H_{\text {int }}$ in baryons is nonzero. Only for particles with $S=T=1.5$ and $S=T=4.4$ is this perturbative energy of order 1 zero, and such particles do not physically exist. Table 4 presents our results as compared to the results from the nonrelativistic method [30] and lattice QCD calculations [31, 32]. As observed, there is a good agreement between the two sets of results. References
$[33,34]$ also determine the spectra of $c$ quark and $b$ quark baryons, and they use the color coulomb plus power and the hyper-Coulomb plus linear potentials, respectively, to solve six-dimensional Schrödinger equation numerically using the Mathematica method.

\section{Isgur-Wise Function and Semileptonic Decays of Baryons}

The IWF may be parameterized in different ways $[17,35$, 36]. In the approximation of infinite heavy quark masses, the form factors $F_{i}, G_{i}(i=1,2,3)$ define the semileptonic transition of $\Lambda_{b}$ related to a particular universal IWF. These form factors appear as

$$
F_{1}\left(q^{2}\right)=G_{1}\left(q^{2}\right)=\xi(\omega), F_{2}=F_{3}=G_{2}=G_{3}=0 .
$$

However, all the models similarly do this at the zero recoil point as Taylor's series as

$$
\xi(y)=1-\rho^{2}(y-1)+c(y-1)^{2}+\cdots,
$$

where $\rho$ is the magnitude of the slope and $c$ is the curvature (convexity parameter) of the Isgur-Wise function at $\omega$ $=1$. The higher terms in the IWF may be negligible since slope and curvature are the most dominant parameters. The Isgur-Wise function for the weak decay of heavy baryons transition in the HCQM can be written as an overlap integral of the baryon wave functions and is of the following form:

$$
\xi(y)=\int_{0}^{\infty} 16 \pi^{2} x^{5}\left|\psi_{\nu, \gamma}(x)\right|^{2} \cos (\rho x) d x
$$

Generally, the overlap integral which involves the final and the initial wave function is used to calculate transition matrix elements. In the above equation, only $\left|\psi_{0,0}(x)\right|^{2}$ comes into the picture instead of the overlap integral of the final and the initial state. This is because we have 
TABle 3: Calculated masses $(\mathrm{MeV})$ and energies $(\mathrm{MeV})$ for baryons.

\begin{tabular}{lccccc}
\hline Baryon & $E_{n}^{1}$ & $E_{n}^{1}+E_{0}$ & $\left(J^{P}, T, S\right)$ & Mass & Mass in PDG [29] \\
\hline$\Omega^{-}(\mathrm{sss})$ & +1.04 & -5.764 & $\left(3 / 2^{+}, 0,-3\right)$ & $1683.44 \pm 24$ & $1672.45 \pm 0.29$ \\
$\Sigma^{-}$(dds) & -0.207 & -8.299 & $\left(1 / 2^{+}, 1,-1\right)$ & $1315.062 \pm 7.30$ & $1321.71 \pm 0.07$ \\
$\Lambda^{0}$ (uds) & -1.154 & -3.8 & $\left(1 / 2^{+}, 0,-1\right)$ & $1135.648 \pm 8.7$ & $1115.68 \pm 0.0006$ \\
$\Xi^{+}$(uus) & -0.552 & -4.852 & $\left(1 / 2^{+}, 1,-1\right)$ & $1172.518 \pm 323$ & $1189.37 \pm 0.07$ \\
$\Xi^{0}$ (uss) & -0.27 & -5.298 & $\left(1 / 2^{+}, 1 / 2,-2\right)$ & $1298.95 \pm 16.37$ & $1314.86 \pm 0.2$ \\
$\Xi^{-}$(dss) & -0.271 & -5.33 & $\left(1 / 2^{+}, 1 / 2,-2\right)$ & $1174.239 \pm 16.375$ & $1197.449 \pm 0.07$ \\
$\Lambda_{c}^{+}$(udc) & -0.743 & -9.993 & $\left(1 / 2^{+}, 0,0\right)$ & $2271.158 \pm 20.65$ & $2286.46 \pm 0.14$ \\
$\Sigma_{c}^{++}$(uuc) & -0.36 & -10.18 & $\left(1 / 2^{+}, 0,-1\right)$ & $3615.132 \pm 20.75$ & $3621.2 \pm 0.14$ \\
$\Xi_{c}^{+}$(usc) & -0.70 & -10.866 & $\left(1 / 2^{+}, 1 / 2,-1\right)$ & $2478.823 \pm 30.875$ & $2467.93 \pm 0.18$ \\
$\Xi_{c}^{0}$ (dsc) & -0.71 & -10.588 & $\left(1 / 2^{+}, 1 / 2,-1\right)$ & $2471.922 \pm 28.325$ & $2470.91 \pm 0.25$ \\
$\Omega_{c}^{0}$ (ssc) & -0.34 & -10.93 & $\left(1 / 2^{+}, 0,-2\right)$ & $2734.96 \pm 36.00$ & $2695.2 \pm 1.7$ \\
$\Xi_{c c}^{++}$(ucc) & -0.72 & -8.98 & $\left(1 / 2^{+}, 1 / 2,0\right)$ & $3615.015 \pm 40.43$ & $3621.2 \pm 0.7$ \\
$\Lambda_{b}^{0}$ (udb) & -1.2 & -13.33 & $\left(1 / 2^{+}, 0,0\right)$ & $5632.395 \pm 5.27$ & $5619.60 \pm 0.17$ \\
$\Xi_{b}^{0}$ (usb) & -0.53 & -12.95 & $\left(1 / 2^{+}, 1 / 2,-1\right)$ & $5797.497 \pm 33.325$ & $5791.9 \pm 0.9$ \\
$\Xi_{b}^{-}$(dsb) & -0.49 & -12.89 & $\left(1 / 2^{+}, 1 / 2,-1\right)$ & $5787.497 \pm 33.325$ & $5797.0 \pm 0.9$ \\
$\Omega_{b}^{-}$(ssb) & 0.362 & -10.519 & $\left(1 / 2^{+}, 0,-2\right)$ & $6060.00 \pm 33$ & $6046.1 \pm 1.7$ \\
\hline
\end{tabular}

TABLE 4: Calculated masses of some heavy baryons.

\begin{tabular}{lccc}
\hline Baryon & $\begin{array}{c}\text { Mass (MeV) } \\
\text { (ours) }\end{array}$ & $\begin{array}{c}\text { Mass (MeV) } \\
\text { (relativistic method } \\
[30])\end{array}$ & $\begin{array}{c}\text { Mass in QCD } \\
\text { lattice [31, 33] }\end{array}$ \\
\hline$\Lambda_{b}$ & $5632.395 \pm 5.27$ & 5620 & 5641 \\
$\Lambda_{c}$ & $2286.46 \pm 0.14$ & 2286 & 2275 \\
$\Omega_{b}$ & $6046.1 \pm 1.70$ & 6064 & 6010 \\
$\Omega_{c}$ & $2695.2 \pm 1.70$ & 2698 & 2677 \\
$\Xi_{b}$ & $5797.49 \pm 33.32$ & 5803 & 5781 \\
$\Xi_{c}$ & $2478.82 \pm 30.87$ & 2470.88 & 2646 \\
$\Xi_{c c}^{++}$ & $3615.01 \pm 40.43$ & 3622.40 & 3640 \\
\hline
\end{tabular}

investigated the Isgur-Wise function near the zero recoil point $\omega=1$, where the four velocities of the baryons before and after transitions are identical.

If $\cos (p x)$ is expanded, then

$$
\cos (p x)=1-\frac{p^{2} x^{2}}{2 !}+\frac{p^{4} x^{4}}{4 !}+\cdots
$$

where $p^{2}=2 m^{2}(\omega-1) \cdot p^{2}$ stands for the square of virtual momentum transfer. Then,

$$
\begin{aligned}
\xi(y)= & \int_{0}^{\infty} 16 \pi^{2} x^{5}\left|\psi_{0,0}(x)\right|^{2} d x-16 \pi^{2} m^{2}(\omega-1) \\
& \times \int_{0}^{\infty} x^{7}\left|\psi_{0,0}(x)\right|^{2} d x+\frac{8}{3} \pi^{2} m^{4}(\omega-1)^{2} \\
& \times \int_{0}^{\infty} x^{9}\left|\psi_{0,0}(x)\right|^{2} d x
\end{aligned}
$$

TABLE 5: Slope and curvature of IWF for some baryons.

\begin{tabular}{lcccccc}
\hline Baryon & $\rho^{2}$ (our) & $\rho^{2}$ (other) & Ref & $c$ (our) & $c$ (other) & Ref. \\
\hline$\Xi_{b}^{0}$ & 1.85 & 1 & {$[37]$} & 0.93 & 1 & {$[37]$} \\
& & 1.5 & {$[38]$} & & 0.88 & {$[39]$} \\
$\Xi_{c}^{+}$ & 1.75 & 1.77 & {$[37]$} & 0.84 & 0.81 & {$[37]$} \\
& & 1.81 & {$[39]$} & & 0.71 & {$[39]$} \\
$\Lambda_{b}^{0}$ & 1.71 & 1.14 & {$[37]$} & 0.42 & 0.56 & {$[37]$} \\
& & 1.61 & {$[38]$} & & 0.3 & {$[38]$} \\
$\Lambda_{c}^{+}$ & & 1.44 & {$[37]$} & & 0.53 & {$[37]$} \\
& \multirow{2}{*}{1.39} & 1.03 & {$[38]$} & 0.49 & 0.25 & {$[38]$} \\
& & 1.47 & {$[39]$} & & 0.47 & {$[39]$} \\
$\Omega_{b}^{+}$ & 2.49 & 2.56 & {$[37]$} & 1.4 & 1.43 & {$[37]$} \\
$\Omega_{c}^{+}$ & 2.2 & 2.25 & {$[37]$} & 1.2 & 1.10 & {$[37]$} \\
\hline
\end{tabular}

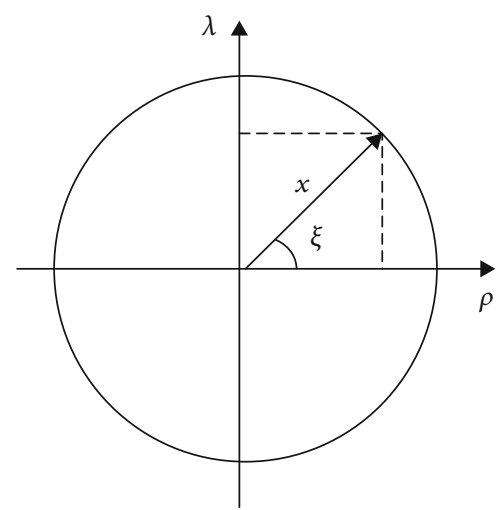

Figure 3: Differential decay width for c baryons. 


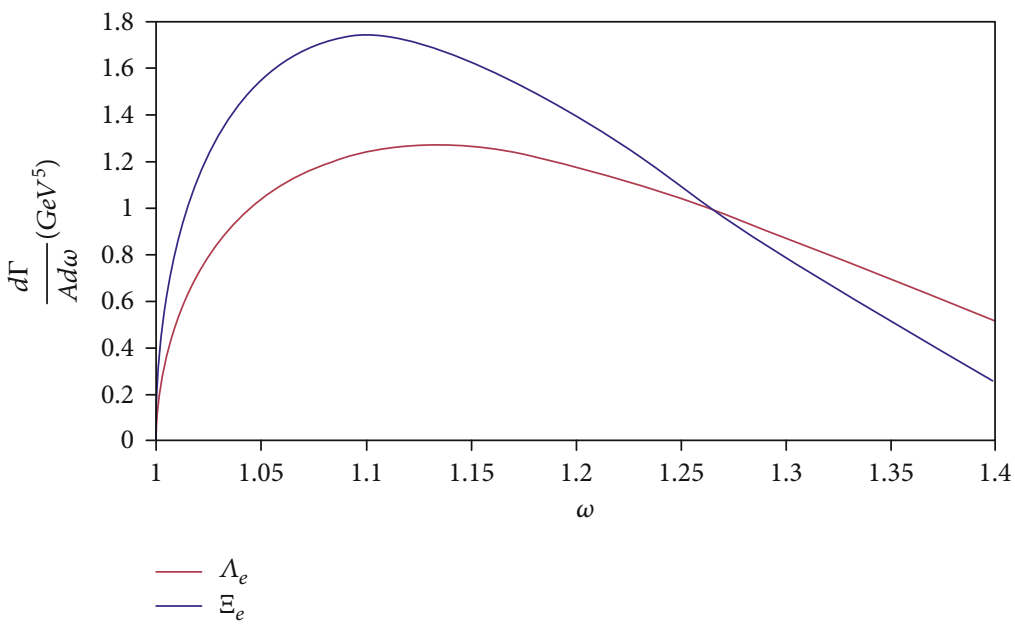

Figure 4: Differential decay width for b baryons.

Comparing equations (30) and (33), we arrive at the slope $\left(\rho^{2}\right)$ and curvature $(c)$ parameters as

$$
\begin{aligned}
& \rho^{2}=16 \pi^{2} m^{2} \int_{0}^{\infty} x^{7}\left|\psi_{0,0}(x)\right|^{2} d x, \\
& c=\frac{8}{3} \pi^{2} m^{4} \int_{0}^{\infty} x^{9}\left|\psi_{0,0}(x)\right|^{2} d x .
\end{aligned}
$$

The Isgur-Wise function mentioned by equation (35) depends on the product of two terms: the first is the square of the modulus of the wave function $\left(\left|\psi_{v, \gamma}(x)\right|^{2}\right)$ and the second is $\cos (p x)$. The value of the cosine term appeared in the Isgur-Wise function becomes 1 , when we put $\omega=1$. And the remaining term $\left(\left|\psi_{v, \gamma}(x)\right|^{2}\right)$ gives $\xi(\omega=1)$, while integrating it for the ground state $(\nu=0, \gamma=0)$ wave function at the zero recoil point. The obtained slope and curvature for $\Xi_{c}^{+}$, $\Xi_{b}^{0}, \Lambda_{b}^{0}, \Lambda_{c}^{+}, \Omega_{b}$, and $\Omega_{c}$ baryons appear in Table 5. The IWF presented in this section is the key to calculating the decay width for heavy baryon transitions. Other works also have employed this method, but they have used different interaction potentials. For instance, [37] uses Cornell potential, [38] uses a Killingbeck potential. Reference [39] makes use of the Hulthen plus linear to describe baryon interactions. However, our findings are well consistent with theirs. In Figure 3, we illustrate the behavior of $d \Gamma / A d \omega$ in terms of $\omega$ for a few baryons in two graphs. For example, for semileptonic decay $\Lambda_{b} \longrightarrow \Lambda_{c}$ transition, we have calculated differential decay width by

$$
\frac{d \Gamma}{d \omega}=\frac{2}{3} m_{2}^{4} m_{1} A \xi^{2}(\omega) \sqrt{\omega^{2}-1}\left[3 \omega\left(\eta+\eta^{-1}\right)-2-4 \omega^{2}\right],
$$

where

$$
A=\frac{G_{F}^{2}}{(2 \pi)^{3}}\left|V_{c b}\right|^{2} B(\Lambda \longrightarrow a b),
$$

where $\eta=m_{\Lambda_{b}} / m_{\Lambda_{c}}$ and $B\left(\Lambda_{c} \longrightarrow a b\right)$ is the branching ratio for the decay $\Lambda_{c} \longrightarrow a\left(1 / 2^{+}\right)+b\left(0^{-}\right)$and $\left|V_{c b}\right|$ stands for the Kobayashi-Maskawa matrix element. Also $m_{1}$ and $m_{2}$ refer to the masses of $\Lambda_{b}$ and $\Lambda_{c}$, respectively. To calculate the total decay width, we have integrated equation (36) over the $\omega$ as

$$
\int_{1}^{\omega_{\max }} \frac{d \Gamma}{d \omega} d \omega=\Gamma
$$

The maximum of the $\omega$ values is calculated from

$$
\omega_{\max }=\frac{m_{\Lambda_{b}}^{2}+m_{\Lambda_{c}}^{2}}{2 m_{\Lambda_{b}} m_{\Lambda_{c}}}
$$

Figures 3 and 4 depict the variations of the differential decay width for a few heavy-baryon decays. As expected, the heavier the baryon, the larger the parameters $\rho^{2}$ and $c$, and the more quickly $d \Gamma / A d \omega$ tends to zero. The semileptonic decay of $\Lambda_{b}^{0} \longrightarrow \Lambda_{c}^{+}$transitions is shown in Figure 5 . In this transition, $b$ quark converts to $c$ quark and a virtual $W$ [40], which becomes a charged lepton and neutrino. Using the decay rates of baryons in Table 6 and integrating equation (36), we obtain function $\Gamma$ and calculate its error. To identify the error of equation (38), we applied the natural logarithm to both sides of equation (38) and then derived from it. By considering each term's error, we determined the absolute error of $\Gamma$. Also from the obtained decay width and decay lifetime of each baryon adopted from PDG, we found the branching ratio values for semileptonic decay. The values obtained for $\Lambda_{b}^{0}$ and $\Lambda_{c}^{+}$comply with PDG values. The branching ratio for other decay channels is not given in PDG, and we indeed propose branching ratio values and present them in the last column of Table 6. Using our calculations for the decay width of $\Lambda_{b}^{0} \longrightarrow \Lambda_{c}^{+} e^{-} v_{e}^{-}$and $\Lambda_{c}^{+} \longrightarrow$ $\Lambda^{0} e^{+} v_{e}^{-}$and the values $\tau_{\Lambda_{b}^{0}}=1.471 \pm 0.009$ and $\tau_{\Lambda_{c}^{+}}=$ $0.2024 \pm 0.0031$ for the $\Lambda_{b}^{0}$ and $\Lambda_{c}^{+}$mean life, we get $\operatorname{Br}\left(\Lambda_{b}^{0}\right.$ $\left.\longrightarrow \Lambda_{c}^{+} e^{-} v_{e}^{-}\right)=5.546$ and $\operatorname{Br}\left(\Lambda_{c}^{+} \longrightarrow \Lambda^{0} e^{+} v_{e}^{-}\right)=2.307$, 


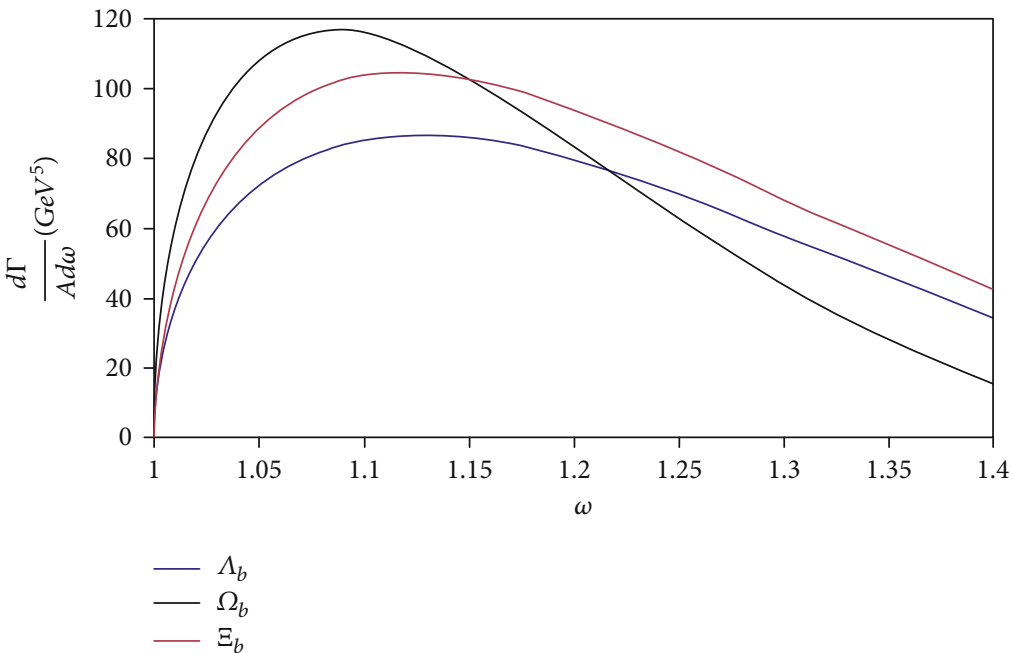

FIGURE 5: Semileptonic decay of $\Lambda_{b}^{0} \longrightarrow \Lambda_{c}^{+}$transitions.

TABLE 6: Rates of some heavy baryon decays and branching ratios calculated by using decay width obtained and experimental mean lives $\tau_{\Xi_{b}^{0}}=1.480 \pm 0.03, \tau_{\Xi_{c}^{+}}=0.456 \pm 0.005, \tau_{\Lambda_{b}^{0}}=1.471 \pm 0.009, \tau_{\Lambda_{c}^{+}}=0.2024 \pm 0.0031, \tau_{\Omega_{b}^{-}}=1.64_{-0.17}^{+0.18}$ (mean life values in 10 $0^{-12} s$ ) [29].

\begin{tabular}{lcccc}
\hline Channel & $\begin{array}{c}\Gamma \text { (ours) } \\
\left(\text { in } 10^{10} s^{-1}\right)\end{array}$ & $\begin{array}{c}\Gamma \text { (others) } \\
\left(\text { in } 10^{10} s^{-1}\right)\end{array}$ & $\begin{array}{c}\text { Branching ratio } \\
(\%) \text { (ours) }\end{array}$ & $\begin{array}{c}\text { Branching ratio } \\
(\%)\end{array}$ \\
\hline$\Xi_{b}^{0} \longrightarrow \Xi_{c}^{+} e^{-} v_{e}^{-}$ & $8.44 \pm 0.422$ & $8.22[37], 7.2[41]$ & $12.493 \pm 0.878$ & - \\
$\Xi_{c}^{+} \longrightarrow \Xi^{-} e^{+} v_{e}^{-}$ & $8.72 \pm 0.018$ & $8.5[41], 8.16[16], 7.4[42]$ & $3.976 \pm 0.052$ & - \\
$\Lambda_{b}^{0} \longrightarrow \Lambda_{c}^{+} e^{-} v_{e}^{-}$ & $3.77 \pm 0.185$ & $3.52[43], 5.12[39], 4.11[44]$ & $5.546 \pm 0.306$ & $6.2_{-1.3}^{+1.4}$ \\
$\Lambda_{c}^{+} \longrightarrow \Lambda^{0} e^{+} v_{e}^{-}$ & $11.4 \pm 0.023$ & $11.2[45], 9.8[41], 13.2[46]$ & $2.307 \pm 0.040$ & $3.63 \pm 0.38 \pm 0.2$ \\
$\Omega_{b}^{-} \longrightarrow \Omega_{c}^{0} e^{-} v_{e}^{-}$ & $1.36 \pm 0.068$ & $1.55[37], 1.87[16]$ & $2.230_{-0.343}^{+0.356}$ & \\
\hline
\end{tabular}

which is acceptable when compared with the result of PDG, which reported $\operatorname{Br}\left(\Lambda_{b}^{0} \longrightarrow \Lambda_{c}^{+} e^{-} v_{e}^{-}\right)=6.2$ and $\operatorname{Br}\left(\Lambda_{c}^{+} \longrightarrow \Lambda^{0} e^{+} \nu_{e}^{-}\right)=3.63$, respectively.

\section{Conclusions}

In this study, we formulated a simple three-body quark model for some baryons. The present model, therefore, has a main SU(6)-invariant part and a perturbative SU(6)-breaking interaction for the splitting within the multiplets. Such a structure may also emerge from lattice QCD calculations. Thus, first we switched off spin and isospin degrees of freedom for the SU(6)-invariant part of the spectrum and considered the baryon as a spin-independent three-body bound system and suggested a semirelativistic quark model for baryons based on the spin-independent three-particle Klein-Gordon equation. We calculated the observable properties of semileptonic decays of bottom and charm baryons, i.e., Isgur-Wise functions, decay rates, and distributions too. Finally, we found the branching ratio values for semileptonic decay. The computed results are compared with other theoretical predictions as well as with the available experimental observations. It can pave the way for further studies, including double heavy quark baryons and their decays. Such stud- ies are important as the current interest in heavy-flavor hadron physics is growing.

\section{Appendix}

\section{A. Review of Nikiforov-Uvarov (NU) Method}

The Schrödinger equation can be converted into a secondorder differential equation as

$$
\sigma^{\prime \prime}(s)+\frac{d^{2} \Psi(s)}{d s^{2}}+\sigma(s) \tilde{\tau}(s) \frac{d \Psi(s)}{d s}+\tilde{\sigma}(s) \Psi(s)=0
$$

where $\sigma(s)$ and $\tilde{\sigma}(s)$ denote polynomials at most of the second degree and $\tilde{\tau}(s)$ is a first-degree polynomial. We use the following form to find the solution:

$$
\Psi(s)=\psi(s) \varphi(s)
$$

By introducing equation (A.2) into equation (A.1), we arrive at

$$
\sigma(s) \frac{d^{2} \varphi(s)}{d s^{2}}+\tau(s) \frac{d \varphi(s)}{d s}+\lambda \varphi(s)=0
$$


where $\varphi(s)$ in terms of Rodriguez formula appears as

$$
\varphi_{n}(s)=\frac{B_{n}}{\rho(s)} \frac{d^{n}}{d s^{n}}\left[\sigma^{n}(s) \rho(s)\right]
$$

The weight function $\rho(s)$ holds in the following formula:

$$
\frac{d \psi(s)}{d s}+\frac{\sigma(s)}{\rho(s)} \frac{d \rho(s)}{d s}=\tau(s)
$$

The other solution factor is defined as

$\pi(s)=\frac{1}{2}\left[\sigma^{\prime}(s)-\tilde{\tau}(s)\right] \pm\left\{\left[\sigma^{\prime}(s)-\tilde{\tau}(s)\right]^{2}-\tilde{\sigma}(s)+k \sigma(s)\right\}^{1 / 2}$

In this method, the polynomial $\pi(s)$ and parameter $k$ are defined as

$$
k=\lambda-\pi^{\prime}(s)
$$

where $\psi(s)$ is defined as

$$
\frac{1}{\psi(s)} \frac{d \psi(s)}{d s}=\frac{\pi(s)}{\psi(s)}
$$

By substituting $k$ into equation (A.6)

$$
\tau(s)=\tilde{\tau}(s)+2 \pi(s)
$$

and $\lambda$ is defined as

$$
\lambda=\lambda_{n}=-n \tau^{\prime}-\frac{\left[n(n-1) \sigma^{\prime \prime}\right]}{2}, \quad n=0,1,2, \cdots
$$

The general form of the Schrödinger equation including any potential is

$$
\frac{d^{2} \psi(s)}{d s^{2}}+\left(\frac{\alpha_{1}-\alpha_{2} s}{s\left(1-\alpha_{3} s\right)}\right) \frac{d \psi(s)}{d s}+\left(\frac{-A s^{2}+B s-C}{s^{2}\left(1-\alpha_{3} s\right)^{2}}\right) \psi(s)=0
$$

Comparing equation (A.1) with equation (A.11), we get the parameters

$$
\tilde{\tau}(s)=\alpha_{1}-\alpha_{2}, \sigma(s)=s\left(1-\alpha_{3} s\right) \tilde{\sigma}(s)=-A s^{2}+B s-C .
$$

Based on the equations, the constant parameters are defined as

$$
\begin{gathered}
\alpha_{4}=\frac{1}{2}\left(1-\alpha_{1}\right), \alpha_{5}=\frac{1}{2}\left(\alpha_{2}-2 \alpha_{3}\right), \\
\alpha_{6}=\alpha_{5}^{2}+A, \alpha_{7}=2 \alpha_{4} \alpha_{5}-B, \\
\alpha_{8}=\alpha_{4}^{2}+C, \alpha_{9}=\alpha_{3} \alpha_{7}+\alpha_{3}^{2} \alpha_{8}+\alpha_{6}, \\
\alpha_{10}=\alpha_{1}+2 \alpha_{4}+2 \sqrt{\alpha_{8}} \quad \alpha_{11}=\alpha_{2}-2 \alpha_{5}+2\left(\sqrt{\alpha_{9}}+\alpha_{3} \sqrt{\alpha_{8}}\right), \\
\alpha_{12}=\alpha_{4}+\sqrt{\alpha_{8}}, \alpha_{13}=\alpha_{5}-\left(\sqrt{\alpha_{9}}+\alpha_{3} \sqrt{\alpha_{8}}\right) .
\end{gathered}
$$

The energy equation is obtained from

$$
\begin{aligned}
& \alpha_{2} n-(2 n+1) \alpha_{5}+(2 n+1)\left(\sqrt{\alpha_{9}}+\alpha_{3} \sqrt{\alpha_{8}}\right)+n(n-1) \alpha_{3} \\
& +\alpha_{7}+2 \alpha_{3} \alpha_{8}+2 \sqrt{\alpha_{8} \alpha_{9}}=0
\end{aligned}
$$

Now we consider the eigenfunctions of the problem with any potential. We obtain the second part of the solution from equation (A.3).

$$
\varphi_{n}(s)=P_{n}^{\left(\alpha_{10}-1,\left(\alpha_{11} / \alpha_{3}\right)-\alpha_{10}-1\right)}\left(1-2 \alpha_{3} s\right) .
$$

From the explicit form of the weight function obtained from equation (A.4), we arrive at

$$
\rho(s)=s^{\alpha_{10}-1}\left(1-\alpha_{3} s\right)^{\left(\alpha_{11} / \alpha_{3}\right)-\alpha_{10}-1} .
$$

$P_{n}^{(\alpha, \beta)}\left(1-2 \alpha_{3} s\right)$ is a Jacobi polynomial. From equation (A.5), we arrive at

$$
\psi(s)=s^{\alpha_{12}}\left(1-\alpha_{3} s\right)^{-\alpha_{12}-\left(\alpha_{13} / \alpha_{3}\right)} .
$$

Then, the general solution $\Psi(s)=\psi(s) \phi(s)$ becomes

$\psi(s)=s^{\alpha_{12}}\left(1-\alpha_{3} s\right)^{-\alpha_{12}-\left(\alpha_{13} / \alpha_{3}\right)} \times P_{n}^{\left(\alpha_{10}-1,\left(\alpha_{11} / \alpha_{3}\right)-\alpha_{10}-1\right)}\left(1-2 \alpha_{3} s\right)$.

\section{Data Availability}

No data were used to support this study.

\section{Conflicts of Interest}

The authors declare that they have no conflicts of interest.

\section{References}

[1] O. Bayrak, G. Kocak, and I. Boztosun, "Anyl-state solutions of the Hulthén potential by the asymptotic iteration method," Journal of Physics A: Mathematical and General, vol. 39, no. 37, pp. 11521-11529, 2006.

[2] B. Gönül, O. Özer, Y. Cançelik, and M. Koçak, "Hamiltonian hierarchy and the Hulthen potential," Physics Letters A, vol. 275 , no. 4, pp. 238-243, 2000. 
[3] R. L. Greene and C. Aldrich, "Variational wave functions for a screened Coulomb potential," Physical Review A, vol. 14, no. 6, pp. 2363-2366, 1976.

[4] W. C. Qiang and S. H. Dong, “Analytical approximations to the solutions of the Manning-Rosen potential with centrifugal term," Physics Letters A, vol. 368, no. 1-2, pp. 13-17, 2007.

[5] S. H. Dong, W. C. Qiang, G. H. Sun, and V. B. Bezerra, “Analytical approximations to thel-wave solutions of the Schrödinger equation with the Eckart potential," Journal of Physics A: Mathematical and Theoretical, vol. 40, no. 34, pp. 1053510540, 2007.

[6] C. Y. Chen, D. S. Sun, and F. L. Lu, “Approximate analytical solutions of Klein-Gordon equation with Hulthen potentials for nonzero angular momentum," Physics Letters A, vol. 370, no. 3-4, pp. 219-221, 2007.

[7] A. D. Alhaidari, "Solution of the relativistic Dirac-Hulthén problem," Journal of Physics A: Mathematical and General, vol. 37, no. 22, pp. 5805-5813, 2004.

[8] G.-F. Wei, C. Y. Long, X. Y. Duan, and S. H. Dong, "Arbitrarylwave scattering state solutions of the Schrödinger equation for the Eckart potential," Physica Scripta, vol. 77, no. 3, article 035001, 2008.

[9] G.-F. Wei, X. Y. Liu, and W. L. Chen, "The relativistic scattering states of the Hulthén potential with an improved new approximate scheme to the centrifugal term," International Journal of Theoretical Physics, vol. 48, no. 6, pp. 1649-1658, 2009.

[10] P. Gonzalez, A. Valcarce, H. Garcilazo, and J. Vijande, "Heavy meson description with a screened potential," Physical Review $D$, vol. 68, no. 3, article 034007, 2003.

[11] B. Silvestre-Brac, "Spectrum and static properties of heavy baryons," Few-Body Systems, vol. 20, no. 1, pp. 1-25, 1996.

[12] M. Radin, S. Babaghodrat, and M. Monemzadeh, "Estimation of heavy baryon masses $\Omega_{c c c}^{++}$and $\Omega_{b b b}^{-}$by solving the Faddeev equation in a three-dimensional approach," Physical Review D, vol. 90, no. 4, article 047701, 2014.

[13] D. Chakraverty, T. De, B. Dutta-Roy, and K. S. Gupta, "Dispersive bounds on the shape of $\Lambda_{b} \longrightarrow \Lambda_{b} l_{\bar{v}_{l}}$ form factors," International Journal of Modern Physics A, vol. 14, no. 15, pp. 23852395, 1999.

[14] S. P. Booth, K. C. Bowler, N. M. Hazel et al., "The Isgur-Wise function from the lattice," Physical Review Letters, vol. 72, no. 4, pp. 462-465, 1994.

[15] A. F. Krutov, O. I. Shro, and V. E. Troitsky, "Isgur-Wise function in a relativistic model of constituent quarks," Physics Letters B, vol. 502, no. 1-4, pp. 140-146, 2001.

[16] M. A. Ivanov, V. E. Lyubovitskij, J. G. Körner, and P. Kroll, "Heavy baryon transitions in a relativistic three-quark model," Physical Review D, vol. 56, no. 1, pp. 348-364, 1997.

[17] H. W. Ke, X. H. Yuan, X. Q. Li, Z. T. Wei, and Y. X. Zhang, " $\Sigma_{b}$ $\longrightarrow \Sigma_{c}$ and $\Omega_{b} \longrightarrow \Omega_{c}$ weak decays in the light-front quark model," Physical Review D, vol. 86, no. 11, article 114005, 2012.

[18] N. A. Viet, "Heavy diquark effective theory and supersymmetry of hadrons containing a single heavy quark," Communications in Physics, vol. 24, no. 1, pp. 35-35, 2014.

[19] D. Ebert, R. N. Faustov, and V. O. Galkin, "Semi-leptonic decays of heavy baryons in the relativistic quark model," Physical Review D, vol. 73, no. 9, article 094002, 2006.

[20] F. Cardarelli and S. Simula, "Isgur-Wise form factors of heavy baryons within a light-front constituent quark model," Physical Review D, vol. 421, no. 1-4, pp. 295-302, 1998.
[21] J. G. Körner, M. Krämer, and D. Pirjol, "Heavy baryons," Progress in Particle and Nuclear Physics, vol. 33, pp. 787-868, 1994.

[22] M. Monemzadeh, N. Tazimi, and P. Sadeghi, "Tetraquarks as diquark-antidiquark bound systems," Physics Letters B, vol. 741, pp. 124-127, 2015.

[23] M. Monemzadeh, M. Hadizadeh, and N. Tazimi, "Identification of the mass and stability interval of strong potential in heavy mesons," International Journal of Theoretical Physics, vol. 50, no. 3, pp. 737-743, 2011.

[24] M. Radin and N. Tazimi, "Quark-antiquark bound state in momentum-helicity representation," Physical Review D, vol. 90, no. 8, article 085020, 2014.

[25] M. Monemzadeh, N. Tazimi, and S. Babaghodrat, "Calculating masses of pentaquarks composed of baryons and mesons," Advances in High Energy Physics, vol. 2016, Article ID 6480926, 4 pages, 2016.

[26] N. Tazimi and A. Ghasempour, "Bound state solutions of three-dimensional Klein-Gordon equation for two model potentials by NU method," Advances in High Energy Physics, vol. 2020, Article ID 2541837, 10 pages, 2020.

[27] B. Patel, A. Majethiya, and P. C. Vinodkumar, "Masses and magnetic moments of triple heavy flavour baryons in hypercentral model," Pramana, vol. 72, no. 4, pp. 679-688, 2009.

[28] M. M. Giannini, E. Santopinto, and A. Vassalo, "An overview of the hypercentral constituent quark model," Progress in Particle and Nuclear Physics, vol. 50, no. 2, pp. 263-272, 2003.

[29] L. Baudis, P. A. Zyla, R. M. Barnett et al., "Review of particle physics," Progress of Theoretical and Experimental Physics, vol. 2020, article 083C01, 2020.

[30] R. N. Faustov and V. O. Galkin, "Heavy baryon spectroscopy in the relativistic quark model," Particles, vol. 3, no. 1, pp. 234-244, 2020.

[31] C. Alexandrou, A. Borrelli, S. Güsken et al., "Beautiful baryons from lattice QCD,” Physics Letters B, vol. 337, no. 3-4, pp. 340346, 1994.

[32] K. Orginos, "Charmed and bottom baryon spectrum from lattice QCD,” eCONF C0906083, vol. 1, 2009.

[33] Z. Shah, K. Thakkar, A. Kumar Rai, and P. C. Vinodkumar, "Excited state mass spectra of singly charmed baryons," The European Physical Journal A, vol. 52, no. 10, pp. 1-25, 2016.

[34] K. Thakkar, Z. Shah, A. K. Rai, and P. C. Vinodkumar, "Excited state mass spectra and Regge trajectories of bottom baryons," Nuclear Physics A, vol. 965, pp. 57-73, 2017.

[35] D. Chakraverty, T. De, B. Dutta-Roy, and K. S. Gupta, "Dispersive bounds on the shape of $\Lambda_{b} \longrightarrow \Lambda_{b} l \bar{v}_{l}$ form factors," International Journal of Modern Physics A, vol. 14, no. 15, pp. 23852395, 1999.

[36] T. Coleman, M. G. Olsson, and S. Veseli, "Semi-leptonic form factors: a model-independent approach," Physical Review D, vol. 63, no. 3, article 032006, 2001.

[37] H. Hassanabadi, S. Rahmani, and S. Zarrinkamar, "Study of heavy-baryon transitions," Physical Review D, vol. 90, no. 7, article 074024, 2014.

[38] H. Hassanabadi, S. Rahmani, and S. Zarrinkamar, "Analysis of the $\Lambda_{b} \longrightarrow \Lambda_{c}$ mode via the Isgur-Wise approach and hyperspherical coordinates," Physical Review D, vol. 89, no. 11, article 114027, 2014.

[39] H. Hassanabadi and S. Rahmani, "Decay rates and branching ratios of $\Xi_{b} \longrightarrow \Xi_{c}$ and $\Lambda_{b} \longrightarrow \Lambda_{c}$ modes," The European Physical Journal Plus, vol. 131, no. 2, pp. 1-10, 2016. 
[40] S. Meinel and G. Rendon, " $\Lambda_{b} \longrightarrow \Lambda_{c}{ }^{*}(2595,2625) l^{-} \bar{v}$ form factors from lattice QCD," Physical Review D, vol. 103, no. 9, article 094516, 2021.

[41] R. Singleton Jr., "Semi-leptonic baryon decays with a heavy quark," Physical Review D, vol. 43, no. 9, pp. 2939-2950, 1991.

[42] H.-Y. Cheng and B. Tseng, "1/M corrections to baryonic form factors in the quark model," Physical Review D, vol. 53, no. 3, pp. 1457-1469, 1996.

[43] K. C. Bowler, R. D. Kenway, L. Lellouch et al., "First lattice study of semi-leptonic decays of $\Lambda_{b}$ and $\Xi_{b}$ baryons," Physical Review D, vol. 57, no. 11, pp. 6948-6974, 1998.

[44] K. Thakkar, "Semi-leptonic transition of $\Lambda_{b}$ b baryon," The European Physical Journal C, vol. 80, no. 10, pp. 1-6, 2020.

[45] R. Perez-Marcial, R. Huerta, A. Garcia, and M. Avila-Aoki, "Predictions for semi-leptonic decays of charm baryons. II. Nonrelativistic and MIT bag quark models," Physical Review D, vol. 40, no. 9, pp. 2955-2966, 1989.

[46] R. S. Marques de Carvalho, F. S. Navarra, M. Nielsen, E. Ferreira, and H. G. Dosch, "Form factors and decay rates for heavy $\Lambda$ semileptonic decays from QCD sum rules," Physical Review D, vol. 60, no. 3, article 034009, 1999. 